\title{
Role of lymph node dissection at the time of open or minimally invasive nephroureterectomy
}

\author{
Andrew G. McIntosh ${ }^{1}$, Eric C. Umbreit ${ }^{1}$, Christopher G. Wood ${ }^{1}$, Surena F. Matin ${ }^{1}$, Jose A. Karam ${ }^{2}$ \\ ${ }^{1}$ Department of Urology, The University of Texas MD Anderson Cancer Center, Houston, TX, USA; ${ }^{2}$ Department of Urology and Translational \\ Molecular Pathology, The University of Texas MD Anderson Cancer Center, Houston, TX, USA \\ Contributions: (I) Conception and design: JA Karam, AG McIntosh, EC Umbreit; (II) Administrative support: JA Karam; (III) Provision of study \\ materials or patients: None; (IV) Collection and assembly of data: AG McIntosh; (V) Data analysis and interpretation: AG McIntosh, JA Karam, SF \\ Matin; (VI) Manuscript writing: All authors; (VII) Final approval of manuscript: All authors. \\ Correspondence to: Jose A. Karam, MD, FACS. Associate Professor, Department of Urology, The University of Texas MD Anderson Cancer Center, \\ 1515 Holcombe Blvd, Unit 1373, Houston, TX 77030, USA. Email: jakaram@mdanderson.org.
}

\begin{abstract}
Unlike urothelial carcinoma of the bladder, there is no guideline-based consensus on whether a lymph node dissection (LND) should be performed at the time of radical nephroureterectomy (RNU) for upper tract urothelial carcinoma (UTUC). Variable lymphatic drainage based on primary tumor location, lack of prospective trials, and difficulties in developing a risk-adapted approach to LND for UTUC are all challenges to the development of an established approach. The UTUC literature consists of an evidence pool that has historically been limited to single-institution series with heterogenous inclusion criteria for LND and variable LND templates. Areas of controversy exist regarding migration to the great vessel LN beds for mid and distal tumors. A lack of template standardization limits the interpretation of studies relative to one another and a lack of uniformity in reporting templates may lead to inaccuracies in the estimation of lymph node metastasis landing sites. Most clinicians agree that there is a staging benefit to LND for UTUC. Although the data is somewhat heterogenous, it demonstrates a prognostic and staging benefit to LND in higher stages of UTUC. Unlike the staging benefits provided by LND for UTUC, the therapeutic benefits are not as clearly established. Several studies have evaluated differences in cancer-specific survival (CSS) and demonstrated LND to be an independent predictor of CSS when compared to patients not undergoing LND. However, this finding is not consistent across all studies and the literature is again limited by inclusion heterogeneity and inconsistent or lack or template-based resections. LND for UTUC at the time of RNU is a safe and feasible procedure that seems to especially benefit patients with muscle-invasive or locally advanced disease. Prospective, randomized studies with strict inclusion criteria and defined anatomic templates are needed to definitely characterize the role of LND for UTUC.
\end{abstract}

Keywords: Lymph node dissection (LND); nephroureterectomy; urothelial carcinoma (UC)

Submitted May 26, 2019. Accepted for publication Nov 15, 2019.

doi: $10.21037 /$ tau.2019.11.34

View this article at: http://dx.doi.org/10.21037/tau.2019.11.34

\section{Introduction}

The standard of care for most cases of invasive upper tract urothelial carcinoma (UTUC) is radical nephroureterectomy (RNU) with bladder cuff excision. In contrast to urothelial carcinoma (UC) of the bladder (1), however, the performance of a regional lymph node (LN) dissection (LND) for UTUC remains controversial. LND for $\mathrm{UC}$ of the bladder is universally accepted by clinicians as an indispensable component of the surgical management of bladder cancer and may offer improvements in oncologic outcomes while allowing more accurate risk stratification of patients (2-4), UTUCs are much more commonly invasive 
at diagnosis ( $60 \%)$ compared to bladder cancer despite LND not being routinely performed for UTUC (5). While the incidence of $\mathrm{LN}$ metastasis $(\mathrm{LNM})(\mathrm{pN}+)$ in UTUC may be as high as $30 \%(6)$, there is significant fluctuation in this incidence with pathologic stage and grade of the primary tumor (7). Moreover, accurate disease staging at initial UTUC evaluation with imaging and ureteroscopy/ biopsy is challenging. As a result, developing risk-adapted criteria to determine which patients ought to receive an LND is challenging. Variable lymphatic drainage from the renal pelvis and ureter and a lack of accepted guideline recommendations further clouds the development of an established approach. With this review, we will summarize the current state of the literature by reviewing the incidence and current rationale for LND and by highlighting the current understanding of lymphatic drainage of UTUC and anatomic templates for LND, the role of LND in staging UTUC, the therapeutic role of LND, and complications and morbidity associated with LND for UTUC.

\section{Materials and methods}

A MEDLINE search was performed utilizing a computerized search engine in order to identify articles pertinent to the role of LND for UTUC. The following search terms were utilized: ("nephroureterectomy" OR ("upper tract" AND "urothelial")) AND ("lymphadenectomy" OR "lymphatic" OR "lymph node dissection"). No restrictions were placed on time period and only English-language articles and articles pertaining to humans were reviewed. Relevant papers were agreed upon by the authors and reviewed in full. If an article contained a reference or citation felt to be relevant, it was reviewed if not already included in the index search.

\section{Results}

\section{Rationale for LND in UTUC}

Non-organ confined UTUC portends a poor prognosis with a 5 -year CSS of $35 \%$ in $\mathrm{N}+$ disease, diminished from $>80 \%(5,8)$. Due to its relative rarity (9), conclusions regarding the impact of LND on UTUC outcomes are not as readily derived from single-institution series as compared to UC of the bladder. LND for invasive bladder cancer has been adopted as a management standard and a full pelvic LND is mandatory at the time of surgical extirpation (10). Beyond the advantages of risk stratification, a thorough and extensive pelvic LND has been shown in retrospective series to improve survival outcomes $(11,12)$ and potentially cure a subset of patients with LNM (2). Additionally, Koppie et al. demonstrated that survival continues to increase as the number of nodes removed increases (4). However, a newly published randomized-controlled trial failed to demonstrate superior recurrence-free survival (RFS) with extended as opposed to limited LND which may call into question the extent of pelvic LND that is required (13).

It is on the basis of the bladder cancer literature that investigators have extrapolated a likely patient population in whom LND for UTUC might provide a staging and/ or therapeutic benefit. Although there are similarities between UC of the bladder and UTUC, LND is not regularly performed at RNU worldwide despite the incidence of LNM ranging from 10-28\% in large multicenter studies $(5,14,15)$. Recently, a Canadian populationbased analysis revealed that only $27 \%$ of surgical specimens contained LNs (16) indicating that adoption of LND in practice is inconsistent and infrequent. This is in part the result of an evidence pool that has historically been limited to single-institution series with heterogenous (or nonexistent) inclusion criteria for LND and variable LND templates (17-20). The Upper Tract Urothelial Carcinoma Collaborative (UTUCC) was developed in response to the lack of high-quality data which has resulted in more recent multi-institutional series investigating the prognostic and therapeutic role of LND in UTUC $(15,21)$. It is important to realize that all of the published studies are limited by a lack of selection criteria and/or significant heterogeneity exists between studies.

\section{Lymphatic drainage sites and anatomic templates in LND for UTUC}

Given the lack of consensus regarding the oncologic benefits of LND for UTUC, clearly defined anatomic templates are needed to provide a framework for high quality prospective trials. Until recently, regional lymphatic drainage for UTUC was defined as the respective renal hilar nodal beds in addition to the para-caval (right side) or para-aortic (left side) LNs for renal pelvis/proximal tumors and intra-pelvic nodes for distal ureteral tumors. This understanding, however, was primarily based upon early mapping studies published in the 1980s $(22,23)$.

Kondo et al. performed a detailed mapping study of 42 patients which revealed wider landing sites for LNM 
than previously understood (24). In this study, $23.2 \%$ of patients had LNM and the authors recommendations were made on the basis of those sites with $>30 \%$ incidence of primary nodal metastasis. In addition to the ipsilateral renal hilar nodes, renal pelvis tumors were mapped to the paracaval/retrocaval nodes for right-sided tumors and the para-aortic nodes for left-sided tumors. The upper twothirds of the ureter similarly drained to the paraaortic nodes on the left and the retrocaval and inter-aortocaval nodes on the right while the lower one-third of both ureters were confined to pelvic LN beds. An updated study $(\mathrm{n}=75)$ expanded these recommendations to include a wider template including inter-aortocaval nodes for right RP tumors and pre-sacral nodes for distal ureteral tumors (25).

The most recent mapping work by Matin $e t$ al. further refined our understanding of LN drainage sites (26). Specifically, in an analysis of 73 patients across multiple institutions, the authors confirmed the migration pattern for renal pelvis and proximal ureter tumors, but also observed cranial migration from mid (62.5-100\%) and distal (16.3$25 \%)$ tumors to paracaval/paraaortic LNs. Notably, outof-field LNM appeared to occur secondarily which may suggest a role for intra-operative frozen section prior to proceeding with extended dissections (Figures 1,2) (27).

Ultimately, the majority of available studies demonstrate anatomical templates of LND for renal pelvis and proximal ureteral tumors involve $\mathrm{LN}$ beds along the great vessels including the renal hilum while distal tumors include intrapelvic nodes. Areas of controversy exist regarding migration to the great vessel $\mathrm{LN}$ beds for mid and distal tumors. A lack of standardization limits the interpretation of studies relative to one another and a lack of uniformity in reporting templates may lead to inaccuracies in the estimation of LNM landing sites.

\section{Role of LND in staging UTUC}

The presence of LNM is a poor prognostic sign associated with a significant decline in survival outcomes (28). Although results are somewhat contradictory, there is a clear staging benefit to LND for UTUC. Historically, much of the available data came from single institution retrospective series suggesting that there was a predictive value of LND $(20,24,29)$. Specifically, these studies demonstrated that pN0 patients had improved cancer-specific survival (CSS) compared to those with $\mathrm{pN}+$ disease (5-year actuarial CSS ranges: $56-85 \%$ vs. $0-39 \%$, respectively). These findings suggest that by establishing accurate staging with LND at the time of RNU, clinicians ought to be able to improve post-operative risk stratification and offer more nuanced patient counseling regarding triage into follow up or adjuvant therapy pathways. Notably, in two of the studies, $\mathrm{pNx}$ status was also found to be an independent predictor of decreased survival when compared to patients who were pN0 $(20,24)$. Other authors, however, did not note any differences in disease-free survival (DFS) or CSS when evaluated by nodal status (30).

More recent multi-institutional analyses with larger patient cohorts have added significant granularity to the literature and may offer more robust conclusions $(14,15$, 31-33). These studies demonstrate a consistently worse prognosis for $\mathrm{pN}+$ patients when compared to $\mathrm{pN} 0$ or $\mathrm{pNx}(14,32,33)$. Indeed, a large population-based study of 2,824 patients demonstrated significant differences in 5-year CSS rates for $\mathrm{pN}+(34 \%)$ vs. $\mathrm{pNx}(78 \%)$ or $\mathrm{pN} 0(81 \%)$ patients $(\mathrm{P}<0.001)$ (32). This was not the case, however, for establishing prognostic differences between $\mathrm{pN} 0$ and $\mathrm{pNx}$ patients. In fact, only two of these multi-institutional series found a significant difference in CSS between pN0 and $\mathrm{pNx}$ disease $(15,31)$. Additionally, Ouzzane et al. found that on multivariable analysis, LN status did not independently predict CSS [pN+vs. pN0: HR 1.6 (95\% CI: 0.8-3.4), $\mathrm{P}=0.1$; pNx vs. pN0: HR 1.14 (95\% CI: 0.7-1.9), $\mathrm{P}=0.6$ ] (32). The staging benefit provided by LND appeared to be most substantial in patients with muscle-invasive and/or locally advanced disease when subset analyses were performed $(15,33,34)$ despite this not being consistent across all available multi-institutional studies (32). Studies that did not show a staging benefit also did not specify dissection templates or extent of anatomic dissection.

The demonstration of a staging benefit to LND for UTUC logically leads to the question of whether there is an optimal quality paradigm under which LND should be performed. Roscigno et al. evaluated whether a minimum number of LNs was necessary to be removed to reliably detect LNM (35). They determined, in a cohort of 551 patients across 13 centers, that when $13 \mathrm{LNs}$ were removed there was a $90 \%$ probability of detecting LNM. Moreover, the removal of $8 \mathrm{LNs}$ conferred a $75 \%$ likelihood of LNM and the removal of 8 LNs was an independent predictor of $\mathrm{LN}$ involvement on multivariate analysis (MVA). In a subsequent study of over 800 patients, Xylinas et al. sought to develop a model to predict the likelihood of false-negative LNM (36). The authors demonstrated that the odds of missing LNM decreased as the number of nodes removed increased. However, they note that for patients 
Dissection templates for renal pelvis and proximal ureter tumors

A

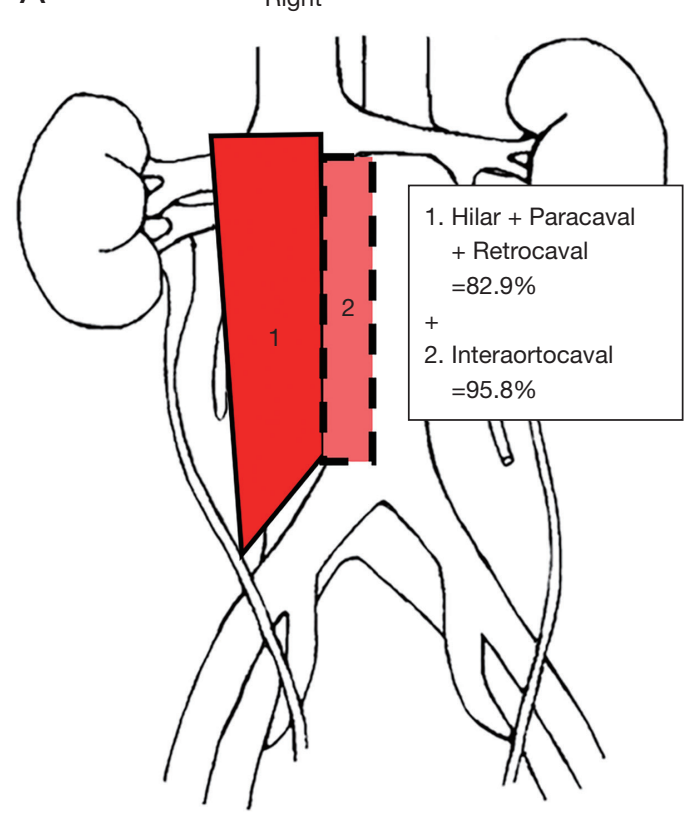

B Left

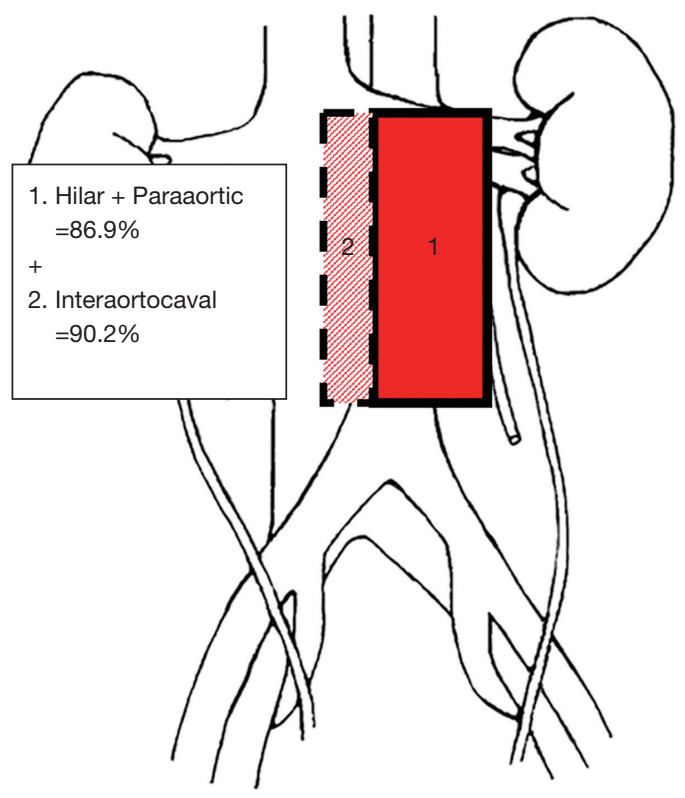

(C) 2019 Surena F. Matin

Figure 1 Consolidated lymph node dissection templates for renal pelvis and proximal ureter tumors. On right side primary hilar, paracaval (anterior precaval and lateral paracaval regions) and retrocaval lymphadenectomy would capture $82.9 \%$ of LNMs (solid outline) while adding interaortocaval lymph node dissection (dashed outline) would increase rate to $95.8 \%$ (A). On left side primary hilar and para-aortic (anterior preaortic and lateral para-aortic regions) lymphadenectomy would capture $86.9 \%$ of LNMs while adding interaortocaval lymph nodes would increase rate to $90.2 \%$ (B) (26) (C) 2019 Surena F. Matin, reprinted with permission). LNMs, lymph node metastases.

with locally advanced disease, even 20 nodes removed did not achieve $95 \%$ accuracy. Conversely, for patients with non-muscle invasive disease and/or lymphovascular invasion, only 2 LNs needed to be removed to achieve $95 \%$ accuracy. These findings suggest that the extent and quality of LND needed may depend on the pathology of the primary tumor. Additionally, Cho et al. (30) subdivided $\mathrm{pN}+$ patients into three groups based on number of $\mathrm{LN}$ removed and found that increasing numbers of $\mathrm{LN}$ removed led to decreased locoregional recurrence on chi-squared test $(\mathrm{P}=-0.009)$ but did not have an impact on CSS $(\mathrm{P}=0.212)$. A critique of these studies is that they use number of LNs removed as an indirect surrogate for resection of the "correct" LNs, rather than dissection of anatomic templates, which may be more relevant than the number removed. Also, it must be noted that there are limitations in the generalizability of recommendations for minimum LNs removed as there is often a chasm between the degree of nodal dissection by the surgeon and nodes examined by the pathologist. The concept of $\mathrm{LN}$ density (percentage of positive LNs) has also been shown to add prognostic value. Herr et al. initially evaluated the concept in the bladder cancer literature by demonstrating that a LN density of $\geq 20 \%$ conferred a decrease in overall survival (OS) from $64 \%$ to $8 \%$ (37). Roscigno et al. also demonstrated LN density to be an independent predictor of CSS (HR 1.81, $\mathrm{P}<0.05$ ) on MVA amongst $\mathrm{pN}+$ patients (20). Similarly, the UTUCC found a LN density of $\geq 30 \%$ to portend higher 5 -year rates of disease recurrence ( $25 \%$ vs. $38 \%$, HR 1.8 , $\mathrm{P}=0.02)$ and higher 5 -year mortality (30\% vs. $48 \%$, HR 1.7 , $\mathrm{P}=0.03)$ in UTUC which may add additional prognostic information for node-positive patients (38).

Taken together, these studies demonstrate a prognostic and staging benefit to LND in higher stages of UTUC, however, without prospective randomized trials with strict inclusion criteria and LND templates, definitive conclusions cannot be drawn, especially given the presence of contradictory results. In general, there appears to be 


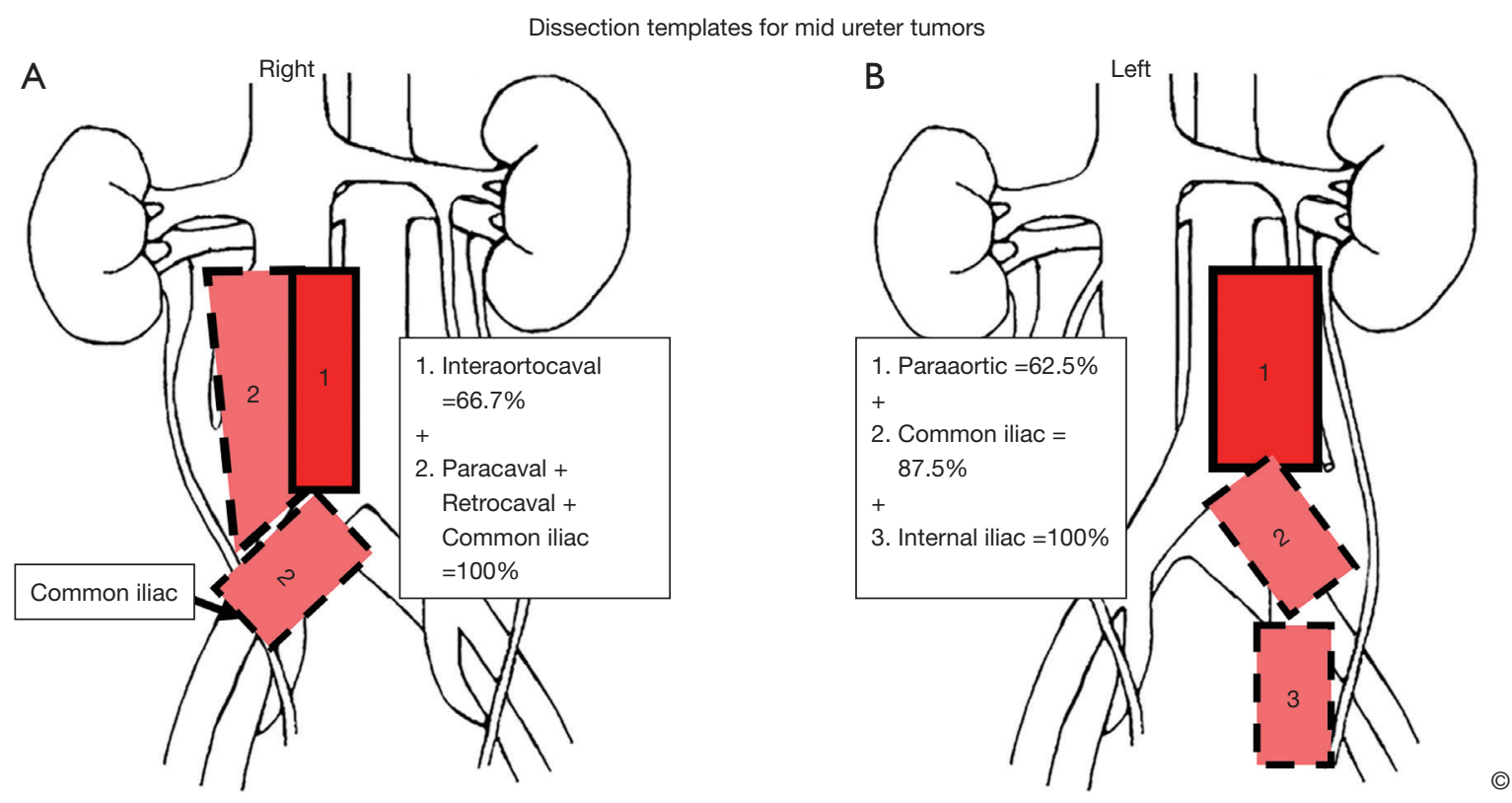

(C) 2019 Surena F. Matin
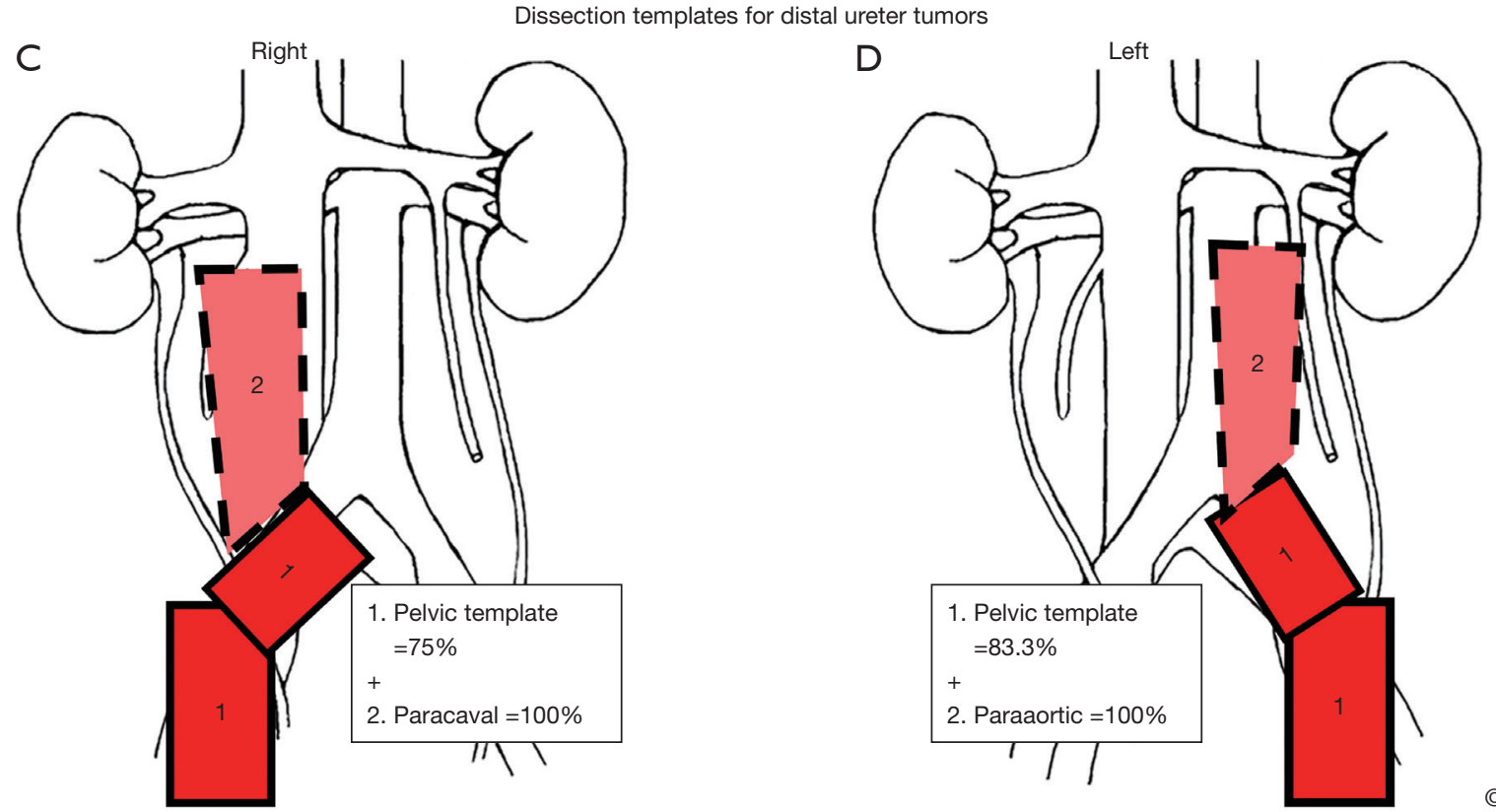

() 2019 Surena F. Matin

Figure 2 Lymph node dissection templates for tumors of mid (A,B) and distal (C,D) ureter developed by combining data from publications by Kondo et al. $(18,24,25)$ That study included three patients with right mid ureter tumors and LNMs to retrocaval and interaortocaval regions (A), and 3 with left mid ureter tumor with LNMs to para-aortic (anterior preaortic and lateral para-aortic) region (B). On right side primary dissection of interaortocaval nodes would capture $66.7 \%$ of possible LNMs (solid outline) while adding paracaval (anterior precaval and lateral paracaval) and retrocaval nodes would remove remaining 33.3\% (dashed outline) (A). No LNMs were identified in common iliac region but they were likely dissected at ureteral resection. On left side para-aortic node primary dissection would remove $62.5 \%$ of LNMs (solid outline), and adding common iliac and internal iliac lymph nodes would increase rate to $100 \%$ (dashed outline) (B). Study by Kondo et al. (18) also included 2 right distal ureter tumors with LNMs to common iliac and obturator regions (C) and 2 left distal ureter tumors with LNMs to common and internal iliac regions (D). Extended pelvic template dissection would capture 75.0\% of LNMs on right side (solid outline) while adding paracaval dissection would increase rate to $100 \%$ (dashed outline) (C). Primary pelvic dissection on left side would capture $83.3 \%$ of LNMs (solid outline) while adding para-aortic dissection would increase rate to 100\% (dashed outline) (D) (26) (C) 2019 Surena F. Matin, reprinted with permission). LNMs, lymph node metastases. 
some consensus amongst clinicians that the staging benefits of LND can be of significant value, particularly in those with higher risk disease (39) (Table 1-summary of staging papers).

\section{Therapeutic role of $L N D$}

Although the role of LND for UTUC is relatively wellestablished for staging, a similar therapeutic role has not been demonstrated to the same degree. In order to assess the therapeutic role, multiple studies have compared outcomes amongst patients undergoing so-called complete, limited, or no LND. A number of studies have evaluated outcomes of patients undergoing LND vs. no LND $(15,17,19,20,30,40)$. With respect to cancer-specific outcomes, several studies have evaluated differences in CSS and demonstrated LND to be an independent predictor of CSS when compared to no LND patients $(15,19,20)$. Roscigno et al., in a single-institutional retrospective analysis found CSS to be longer in pN0 compared to pNx patient $(73 \%$ vs. $48 \%, \mathrm{P}<0.001)$ but no difference between $\mathrm{pNx}$ and $\mathrm{pN}+$ patients (20). In a subsequent multiinstitutional analysis of over 1,100 patients, $\mathrm{pNx}$ status was associated with worse CSS outcomes in patients with pT2-T4 disease only (15) consistent with an earlier, singleinstitution study (19). Again, these studies did not routinely specify if correct anatomic templates were dissected in all cases. Several of these studies do offer descriptions of templates in the study methodology, although they are retrospective and often include multiple surgeons, raising the question of how a strict template can be defined and adhered to in such a design $(17,19,20)$. Conversely, Kondo et al. demonstrated a 3-year CSS rate of $89.8 \%$ for patients undergoing LND compared to $51.7 \%$ for those undergoing no $\mathrm{LND}(\mathrm{HR}=0.23, \mathrm{P}=0.01)$ in a subset analysis of muscleinvasive renal pelvis UTUC patients in whom dissection templates were pre-defined. Conversely, no statistically significant difference in the ureteral tumor subgroup (3-year CSS: $54.2 \%$ vs. $71.7 \%$; HR $=0.99, \mathrm{P}=0.99$ ) was present (40). In these cases, however, the LND templates included only the templates; given the data on cranial migration (26), it is possible that the negative finding is related to incomplete removal of the relevant nodes. Similarly, Cho et al. evaluated CSS outcomes in pT2 UTUC patients undergoing LND and reported no difference in CSS in the absence of defined templates (30).

Several authors have also evaluated OS outcomes in cohorts comparing complete LND (CLND) to no LND
$(17,19,40)$. Patients undergoing LND had superior OS outcomes in studies by Kondo et al. (median OS $86 \%$ vs. $48 \%$, $\mathrm{P}=0.01$ ), which defined the templates of dissection (40) and Brausi et al. (median OS 52.5 vs. 21.2 months) found LND status to be an independent predictor OS $(\mathrm{P}=0.004)(19)$. Miyake et al. also demonstrated a significant difference in 1-, 3-, and 5-year OS but only amongst those patients with no evidence of LNM (100\%, 93\%, and 86\% vs. $79 \%$, $65 \%$, and $50 \%$, respectively, $\mathrm{P}<0.05)(17)$. The authors of this study do note defined anatomic templates for LND, though it is again difficult to imagine strict adherence to the template in a retrospective study such as this one. Finally, RFS was assessed in two single-institutional studies both demonstrating a statistically improved actuarial 5-year RFS in the LND group (64\% \& 64\%) compared to the no LND group (37\% \& 46.3\%) (19,20). One study noted a prolonged time to recurrence from 18.5 to 51.2 months for the LND group (19).

Three studies by Kondo et al. across two institutions evaluated CLND compared to incomplete LND (ILND) and no LND, with the definition of completeness based on their anatomic templates $(18,41,42)$. None of the three studies reported OS. The first, a retrospective singleinstitution series of 169 patients (1989-2006) in which $26.6 \%$ underwent CLND and ILND in 21.3\%. CSS did not differ amongst the groups when assessed across all patients. However, when patients with pT3 stage or higher were isolated CSS was dependent on the extent of LND (CLND superior to no LND but no difference between CLND and ILND) and CLND was a predictive of CSS on MVA ( $\mathrm{P}=0.009)$ (18). An updated analysis of an expanded cohort through 2011 subsequently revealed a survival advantage of CLND over ILND (25). Five - year CSS in the patients with $\mathrm{pT} 2$ or higher $(\mathrm{P}=0.03)$ and $\mathrm{pT} 3$ or higher $(\mathrm{P}=0.01)$ was $77.9 \%$ and $73.2 \%$ in the patients undergoing CLND, but just $54.0 \%$ and $43.7 \%$ in those in the ILND group and $59.0 \%$ and $47.3 \%$ in those with no LND. The same group performed an analysis of $\geq \mathrm{pT} 2, \mathrm{cN} 0, \mathrm{cM} 0$ patients and found a significant CSS benefit to CLND over ILND (14\% vs. 29\%, P=0.04) (41). In this cohort, the ILND group had more patients with $\mathrm{pN}+$ disease $(22 \% v s$. $15 \%$ ) and less patients who received adjuvant chemotherapy which may partially explain some of these outcomes. In a prospective study, these authors also found CLND to be an independent predictor of 2- and 5-year CSS and regional-node RFS compared to ILND and No LND in renal pelvis tumors (42). It is likely that the suggestion of a benefit isolated to renal pelvis tumors and not lower 


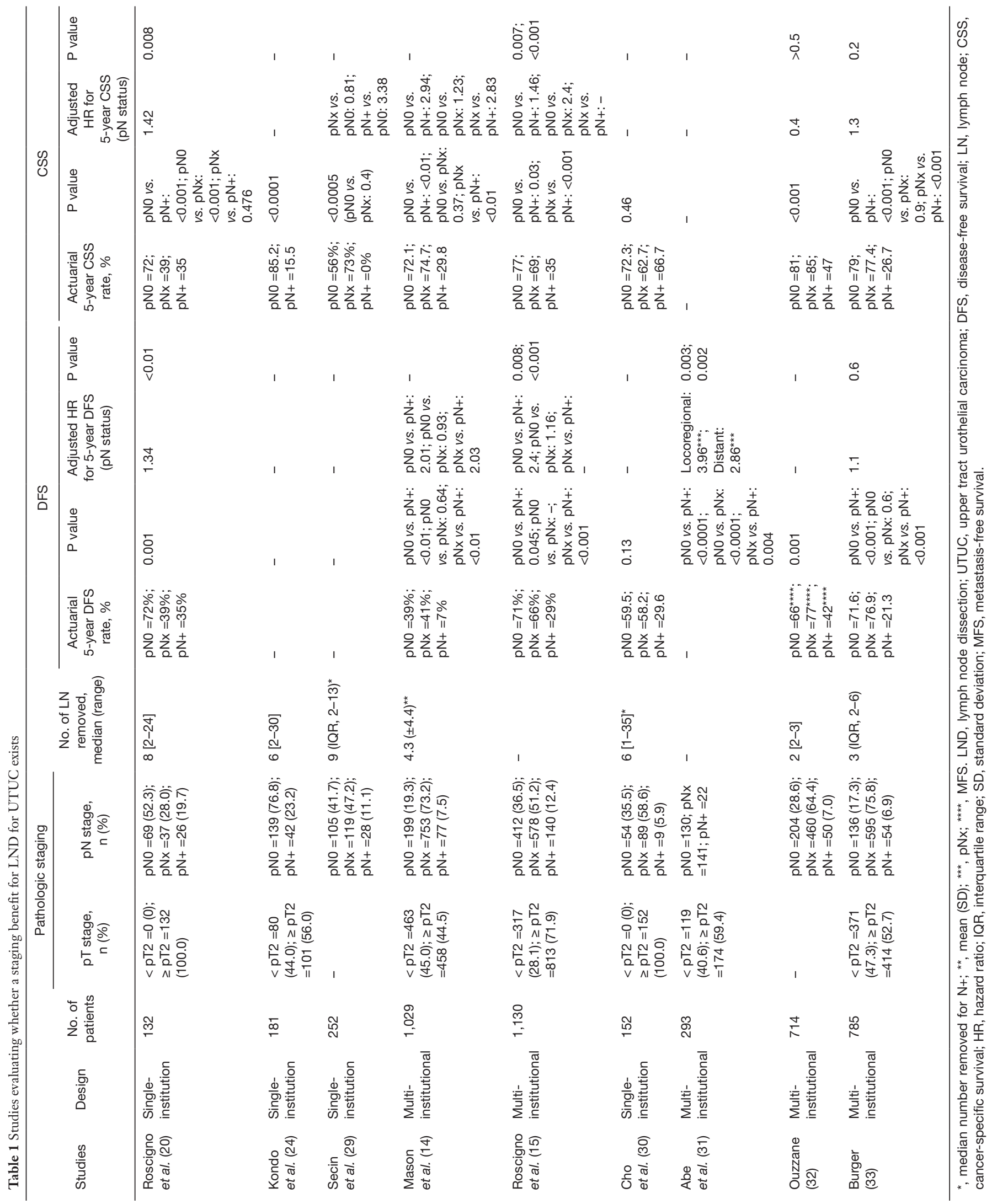


ureteral tumors, however, is due to incorrect or inconsistent templates for these lower tumors and limitations in the study design. Finally, a population study by Lughezzani $e t$ al. demonstrates no therapeutic benefit to LND ( $\mathrm{pNx} v s$. $\mathrm{pN} 0$ CSS, HR 0.99, $\mathrm{P}=0.9$ ) (34) and a large retrospective multiinstitutional study similarly found no CSS or RFS benefit to LND; nor did LND predict RFS or CSS on MVA (33). However, none of these studies defined the templates of dissection (Table 2-summary of therapeutic role of LND).

Accurate pre-operative staging assessment would further improve our ability to discern which patients might benefit most from LND, however this remains a challenge due to limitations with conventional pre-operative evaluation paradigms such as imaging (43) and/or ureteroscopic biopsies $(8,44)$. The UTUCC Group have attempted to address this concern by establishing a nomogram that predicts non-organ confined disease with $>76 \%$ accuracy based on tumor grade, architecture and location (45). More recently, Petros et al. found that a pre-operative nomogram incorporating clinical stage, tumor grade at ureteroscopic biopsy, tumor architecture, and serum hemoglobin level combined to predict high-risk, non-organ confined disease with $82 \%$ accuracy (46). Currently this nomogram is increasingly used at our center to help guide triage to neoadjuvant chemotherapy and LND.

\section{Number of LNs removed vs. dissection templates}

Another remaining question is whether it is the number nodes which should be removed or dissecting in the correct anatomic templates in order to optimize either the staging or therapeutic benefits of LND for UTUC. A singleinstitution series by Roscigno et al. found increasing number of $\mathrm{LN}$ removed to be an independent predictor of DFS and CSS, although not when limited to $\mathrm{pN}+$ patients (20). A subsequent multi-institutional analysis demonstrated that although number of $\mathrm{LN}$ removed was not associated with cancer-specific mortality (CSM) across all patients, CSM seems to improve with the number of LNs removed in the subgroup of $\mathrm{pN} 0$ patients (21). In a similar analysis, increasing number of LNs removed improved DFS (HR 0.94, $\mathrm{P}=0.009)$ and CSS (HR 0.934, $\mathrm{P}=0.015)(15)$. These findings are somewhat refuted by Kondo et al. who found that despite improved survival outcomes for CLND compared to ILND there was no difference with respect to number of nodes removed (41). This speaks to the fact that when anatomical templates were strictly defined, as in this study, it was performing a complete template-based resection that provided a survival benefit. That is, the finding that a higher number of LNs is associated with an oncologic benefit may just be a surrogate for performing something closer to a true template-based resection. These findings highlight the importance of designing future prospective trials with strictly defined anatomical templates.

\section{Complications and morbidity associated with LND for UTUC}

Despite the relatively well-supported staging benefits and growing body of literature tending towards the therapeutic benefits of LND for UTUC, there remains a lack of consensus in the urologist community at-large. This may be, at least in part, due to concerns about perioperative morbidity surrounding LND. In general, however, the safety and feasibility of LND for UTUC is well supported in the literature. Kondo et al. retrospectively compared CLND, ILND, and no LND operative time, estimated blood loss, and hospital length of stay and found them to be similar amongst the groups (25). Similarly, while complications overall were higher in the CLND group, the incidence of major complications were similar (and had a very low incidence) suggesting only an increase in minor complications with more extensive LND. The safety profile of LND for UTUC has also been prospectively validated in two small studies $(47,48)$. Although post-operative complication rates did range between $16 \%$ and $45 \%$, only one major complication occurred across both studies directly related to the LND (a chylous lymphatic leak that required operative intervention). Four intra-operative complications were reported across the 65 patients in both trials $(6.2 \%)$ - two bowel injuries (immediately repaired without subsequent complication), a vascular injury, and a pancreatic injury (recognized and repaired). None had significant resultant sequelae.

An additional are of controversy exists as to whether clinicians can adequately perform LND laparoscopically. A retrospective analysis from MD Anderson Cancer Center demonstrated the feasibility and oncologic efficacy of laparoscopic LND (49). The authors evaluated open $(\mathrm{n}=106) v s$. laparoscopic $(\mathrm{n}=28) \mathrm{RNU}$ plus LND and found that median nodes removed was higher in the laparoscopic group (6 vs. 3, $\mathrm{P}=0.01$ ) while number of positive $\mathrm{LN}$ and LN density were not different. Abe et al. also prospectively validated the feasibility of laparoscopic LND in a small 


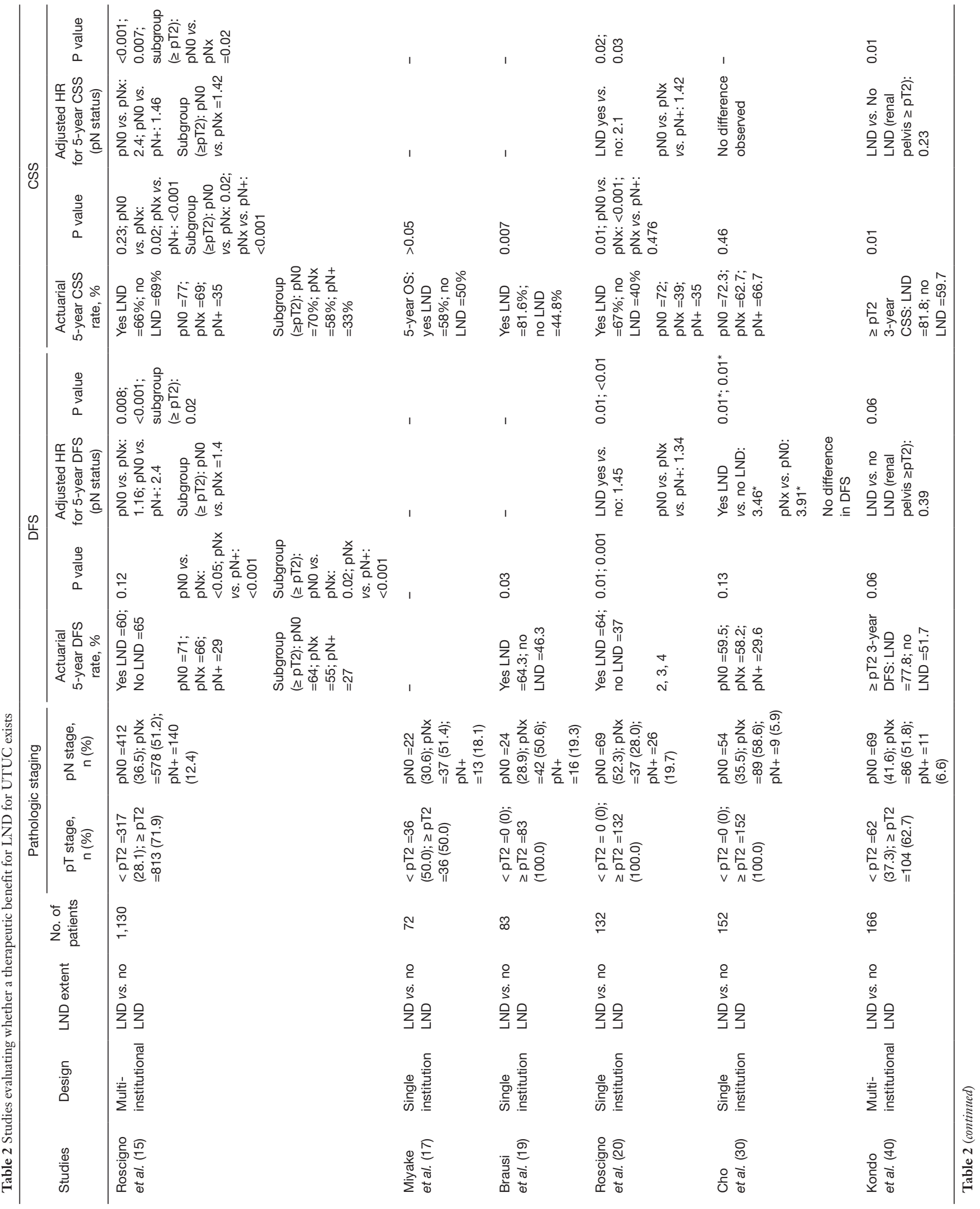




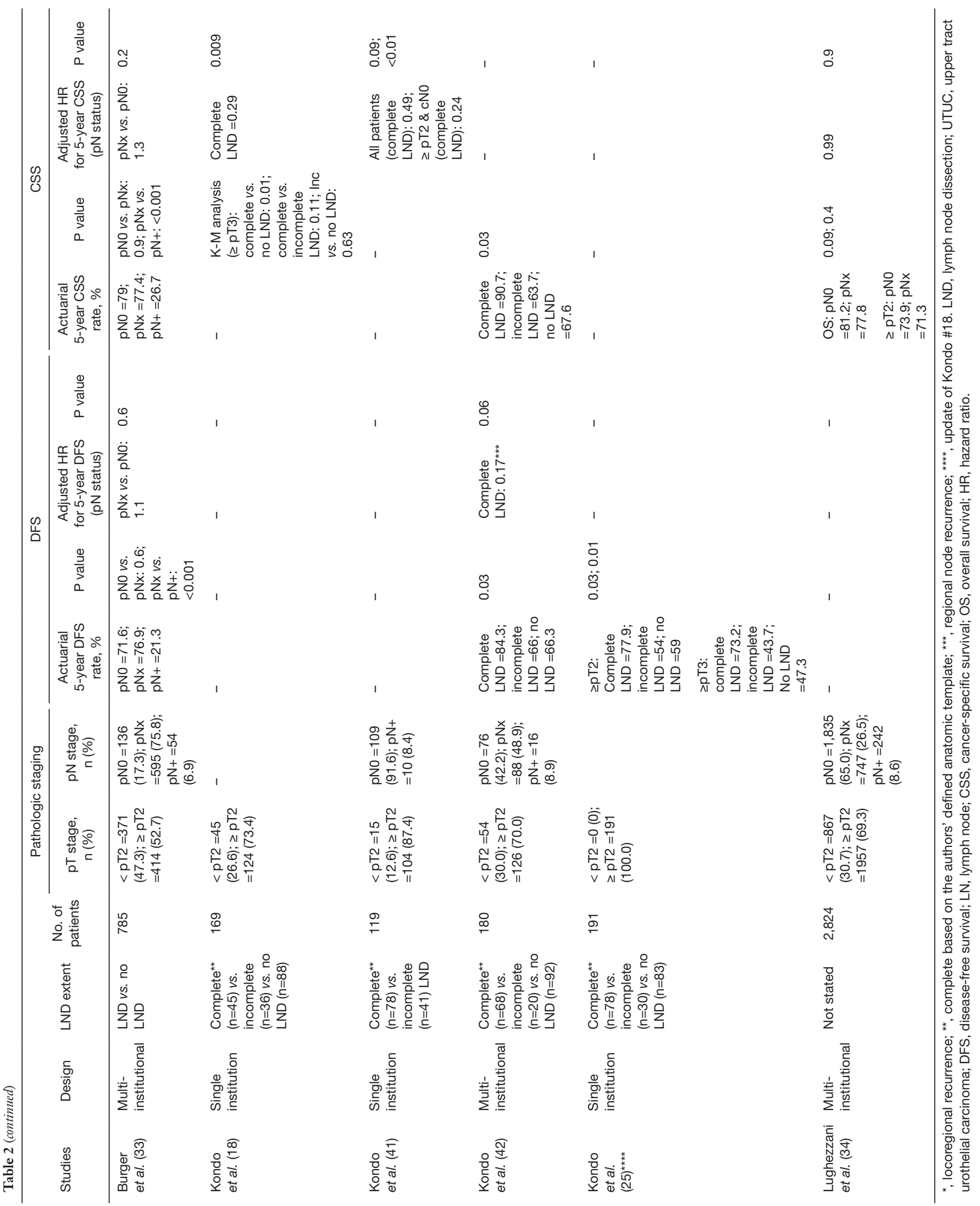


45 patient study (48). A follow up study by MD Anderson investigators demonstrated even higher $\mathrm{LN}$ yields with a robotic vs. laparoscopic RNU approach (50).

\section{Conclusions}

Although the available evidence is primarily limited to retrospective cohorts and a few prospective studies, there is a strong tendency in the literature to support the staging role of LND for UTUC. Similarly, there is a general trend towards the therapeutic benefits of the procedure, although the evidence is less robust and definitive conclusions cannot be drawn. LND for UTUC at the time of RNU is a safe and feasible procedure that may especially benefit patients with muscle-invasive or locally advanced disease. Prospective, randomized studies with strict inclusion criteria and defined anatomic templates are needed to definitely characterize the role of LND for UTUC.

\section{Acknowledgments}

Funding: None.

\section{Footnote}

Provenance and Peer Review: This article was commissioned by the Guest Editors (Marc C. Smaldone and Jeffrey J. Tomaszewski) for the series "Controversies in Minimally Invasive Urologic Oncology” published in Translational Andrology and Urology. The article has undergone external peer review.

Conflicts of Interest: All authors have completed the ICMJE uniform disclosure form (available at http://dx.doi. org/10.21037/tau.2019.11.34). The series "Controversies in Minimally Invasive Urologic Oncology” was commissioned by the editorial office without any funding or sponsorship. Dr. SF Matin is a consultant for QED therapeutics and on the scientific advisory board for TARIS Bio. The authors have no other conflicts of interest to declare.

Ethical Statement: The authors are accountable for all aspects of the work in ensuring that questions related to the accuracy or integrity of any part of the work are appropriately investigated and resolved.

Open Access Statement: This is an Open Access article distributed in accordance with the Creative Commons
Attribution-NonCommercial-NoDerivs 4.0 International License (CC BY-NC-ND 4.0), which permits the noncommercial replication and distribution of the article with the strict proviso that no changes or edits are made and the original work is properly cited (including links to both the formal publication through the relevant DOI and the license). See: https://creativecommons.org/licenses/by-nc-nd/4.0/.

\section{References}

1 Perera M, McGrath S, Sengupta S, et al. Pelvic lymph node dissection during radical cystectomy for muscleinvasive bladder cancer. Nat Rev Urol 2018;15:686-92.

2. Karl A, Carroll PR, Gschwend JE, et al. The impact of lymphadenectomy and lymph node metastasis on the outcomes of radical cystectomy for bladder cancer. Eur Urol 2009;55:826-35.

3. Abdel-Latif M, Abol-Enein H, El-Baz M, et al. Nodal involvement in bladder cancer cases treated with radical cystectomy: incidence and prognosis. J Urol 2004;172:85-9.

4. Koppie TM, Vickers AJ, Vora K, et al. Standardization of pelvic lymphadenectomy performed at radical cystectomy: can we establish a minimum number of lymph nodes that should be removed? Cancer 2006;107:2368-74.

5. Margulis V, Shariat SF, Matin SF, et al. Outcomes of radical nephroureterectomy: a series from the Upper Tract Urothelial Carcinoma Collaboration. Cancer 2009;115:1224-33.

6. Zigeuner R, Pummer K. Urothelial carcinoma of the upper urinary tract: surgical approach and prognostic factors. Eur Urol 2008;53:720-31.

7. Roscigno $M$, Brausi $M$, Heidenreich A, et al. Lymphadenectomy at the time of nephroureterectomy for upper tract urothelial cancer. Eur Urol 2011;60:776-83.

8. Raman JD, Scherr DS. Management of patients with upper urinary tract transitional cell carcinoma. Nat Clin Pract Urol 2007;4:432-43.

9. Roupret M, Babjuk M, Comperat E, et al. European Association of Urology Guidelines on Upper Urinary Tract Urothelial Cell Carcinoma: 2015 Update. Eur Urol 2015;68:868-79.

10. Chang SS, Bochner BH, Chou R, et al. Treatment of Nonmetastatic Muscle-Invasive Bladder Cancer: American Urological Association/American Society of Clinical Oncology/American Society for Radiation Oncology/ Society of Urologic Oncology Clinical Practice Guideline Summary. J Oncol Pract 2017;13:621-5. 
11. Leissner J, Hohenfellner R, Thuroff JW, et al. Lymphadenectomy in patients with transitional cell carcinoma of the urinary bladder; significance for staging and prognosis. BJU Int 2000;85:817-23.

12. Herr HW, Bochner BH, Dalbagni G, et al. Impact of the number of lymph nodes retrieved on outcome in patients with muscle invasive bladder cancer. J Urol 2002;167:1295-8.

13. Gschwend JE, Heck MM, Lehmann J, et al. Extended Versus Limited Lymph Node Dissection in Bladder Cancer Patients Undergoing Radical Cystectomy: Survival Results from a Prospective, Randomized Trial. Eur Urol 2019;75:604-11.

14. Mason RJ, Kassouf W, Bell DG, et al. The contemporary role of lymph node dissection during nephroureterectomy in the management of upper urinary tract urothelial carcinoma: the Canadian experience. Urology 2012;79:840-5.

15. Roscigno M, Shariat SF, Margulis V, et al. Impact of lymph node dissection on cancer specific survival in patients with upper tract urothelial carcinoma treated with radical nephroureterectomy. J Urol 2009;181:2482-9.

16. Abouassaly R, Alibhai SM, Shah N, et al. Troubling outcomes from population-level analysis of surgery for upper tract urothelial carcinoma. Urology 2010;76:895-901.

17. Miyake H, Hara I, Gohji K, et al. The significance of lymphadenectomy in transitional cell carcinoma of the upper urinary tract. Br J Urol 1998;82:494-8.

18. Kondo T, Nakazawa H, Ito F, et al. Impact of the extent of regional lymphadenectomy on the survival of patients with urothelial carcinoma of the upper urinary tract. J Urol 2007;178:1212-7; discussion 1217.

19. Brausi MA, Gavioli M, De Luca G, et al. Retroperitoneal lymph node dissection (RPLD) in conjunction with nephroureterectomy in the treatment of infiltrative transitional cell carcinoma (TCC) of the upper urinary tract: impact on survival. Eur Urol 2007;52:1414-8.

20. Roscigno M, Cozzarini C, Bertini R, et al. Prognostic value of lymph node dissection in patients with muscleinvasive transitional cell carcinoma of the upper urinary tract. Eur Urol 2008;53:794-802.

21. Roscigno M, Shariat SF, Margulis V, et al. The extent of lymphadenectomy seems to be associated with better survival in patients with nonmetastatic upper-tract urothelial carcinoma: how many lymph nodes should be removed? Eur Urol 2009;56:512-8.

22. McCarron JP Jr, Chasko SB, Gray GF Jr. Systematic mapping of nephroureterectomy specimens removed for urothelial cancer: pathological findings and clinical correlations. J Urol 1982;128:243-6.

23. Akaza H, Koiso K, Niijima T. Clinical evaluation of urothelial tumors of the renal pelvis and ureter based on a new classification system. Cancer 1987;59:1369-75.

24. Kondo T, Nakazawa H, Ito F, et al. Primary site and incidence of lymph node metastases in urothelial carcinoma of upper urinary tract. Urology 2007;69:265-9.

25. Kondo T, Tanabe K. Role of lymphadenectomy in the management of urothelial carcinoma of the bladder and the upper urinary tract. Int J Urol 2012;19:710-21.

26. Matin SF, Sfakianos JP, Espiritu PN, et al. Patterns of Lymphatic Metastases in Upper Tract Urothelial Carcinoma and Proposed Dissection Templates. J Urol 2015;194:1567-74.

27. Campi R, Minervini A, Mari A, et al. Anatomical templates of lymph node dissection for upper tract urothelial carcinoma: a systematic review of the literature. Expert Rev Anticancer Ther 2017;17:235-46.

28. Novara G, De Marco V, Gottardo F, et al. Independent predictors of cancer-specific survival in transitional cell carcinoma of the upper urinary tract: multi-institutional dataset from 3 European centers. Cancer 2007;110:1715-22.

29. Secin FP, Koppie TM, Salamanca JI, et al. Evaluation of regional lymph node dissection in patients with upper urinary tract urothelial cancer. Int J Urol 2007;14:26-32.

30. Cho KS, Choi HM, Koo K, et al. Clinical significance of lymph node dissection in patients with muscle-invasive upper urinary tract transitional cell carcinoma treated with nephroureterectomy. J Korean Med Sci 2009;24:674-8.

31. Abe T, Shinohara N, Muranaka M, et al. Role of lymph node dissection in the treatment of urothelial carcinoma of the upper urinary tract: multi-institutional relapse analysis and immunohistochemical re-evaluation of negative lymph nodes. Eur J Surg Oncol 2010;36:1085-91.

32. Ouzzane A, Colin P, Ghoneim TP, et al. The impact of lymph node status and features on oncological outcomes in urothelial carcinoma of the upper urinary tract (UTUC) treated by nephroureterectomy. World J Urol 2013;31:189-97.

33. Burger M, Shariat SF, Fritsche HM, et al. No overt influence of lymphadenectomy on cancer-specific survival in organ-confined versus locally advanced upper urinary tract urothelial carcinoma undergoing radical nephroureterectomy: a retrospective international, multiinstitutional study. World J Urol 2011;29:465-72.

34. Lughezzani G, Jeldres C, Isbarn H, et al. A critical appraisal of the value of lymph node dissection at 
nephroureterectomy for upper tract urothelial carcinoma. Urology 2010;75:118-24.

35. Roscigno M, Shariat SF, Freschi M, et al. Assessment of the minimum number of lymph nodes needed to detect lymph node invasion at radical nephroureterectomy in patients with upper tract urothelial cancer. Urology 2009;74:1070-4.

36. Xylinas E, Rink M, Margulis V, et al. Prediction of true nodal status in patients with pathological lymph node negative upper tract urothelial carcinoma at radical nephroureterectomy. J Urol 2013;189:468-73.

37. Herr HW. Superiority of ratio based lymph node staging for bladder cancer. J Urol 2003;169:943-5.

38. Bolenz C, Shariat SF, Fernandez MI, et al. Risk stratification of patients with nodal involvement in upper tract urothelial carcinoma: value of lymph-node density. BJU Int 2009;103:302-6.

39. Seisen T, Shariat SF, Cussenot O, et al. Contemporary role of lymph node dissection at the time of radical nephroureterectomy for upper tract urothelial carcinoma. World J Urol 2017;35:535-48.

40. Kondo T, Hara I, Takagi T, et al. Template-based lymphadenectomy in urothelial carcinoma of the renal pelvis: a prospective study. Int J Urol 2014;21:453-9.

41. Kondo T, Hashimoto Y, Kobayashi H, et al. Templatebased lymphadenectomy in urothelial carcinoma of the upper urinary tract: impact on patient survival. Int J Urol 2010;17:848-54.

42. Kondo T, Hara I, Takagi T, et al. Possible role of templatebased lymphadenectomy in reducing the risk of regional node recurrence after nephroureterectomy in patients with renal pelvic cancer. Jpn J Clin Oncol 2014;44:1233-8.

43. Scolieri MJ, Paik ML, Brown SL, et al. Limitations of

Cite this article as: McIntosh AG, Umbreit EC, Wood CG, Matin SF, Karam JA. Role of lymph node dissection at the time of open or minimally invasive nephroureterectomy. Transl Androl Urol 2021;10(5):2233-2245. doi: 10.21037/tau.2019.11.34 computed tomography in the preoperative staging of upper tract urothelial carcinoma. Urology 2000;56:930-4.

44. Tavora F, Fajardo DA, Lee TK, et al. Small endoscopic biopsies of the ureter and renal pelvis: pathologic pitfalls. Am J Surg Pathol 2009;33:1540-6.

45. Margulis V, Youssef RF, Karakiewicz PI, et al. Preoperative multivariable prognostic model for prediction of nonorgan confined urothelial carcinoma of the upper urinary tract. J Urol 2010;184:453-8.

46. Petros FG, Qiao W, Singla N, et al. Preoperative multiplex nomogram for prediction of high-risk nonorgan-confined upper-tract urothelial carcinoma. Urol Oncol 2019;37:292. e1-292.e9.

47. Rao SR, Correa JJ, Sexton WJ, et al. Prospective clinical trial of the feasibility and safety of modified retroperitoneal lymph node dissection at time of nephroureterectomy for upper tract urothelial carcinoma. BJU Int 2012;110:E475-80.

48. Abe T, Takada N, Matsumoto R, et al. Outcome of regional lymphadenectomy in accordance with primary tumor location on laparoscopic nephroureterectomy for urothelial carcinoma of the upper urinary tract: a prospective study. J Endourol 2015;29:304-9.

49. Busby JE, Brown GA, Matin SF. Comparing lymphadenectomy during radical nephroureterectomy: open versus laparoscopic. Urology 2008;71:413-6.

50. Melquist JJ, Redrow G, Delacroix S, et al. Comparison of Single-docking Robotic-assisted and Traditional Laparoscopy for Retroperitoneal Lymph Node Dissection During Nephroureterectomy With Bladder Cuff Excision for Upper-tract Urothelial Carcinoma. Urology 2016;87:216-23. 